\title{
Structure et dynamisme de la forêt
}

\author{
Jean-Louis Guillaumet (') \\ Francis Kahn (')
}

\begin{abstract}
Résumé
Les auteurs comparent la struciure de la végétation de deux toposéquences choisies dans les bassins versants de la ZF-C2. Pour ce faire, ils utilisent une méthọde de description morpho-structurale de la végétation dont ils rappellent l'essentiel. Ils discutent des variations selon la position sur la toposéquence et de leurs causes. Ces résultats sont comparés à deux autres séquences. Répartition en classes de diamètres, aire basale sont interprétées en fonction du cycle silvigénétique. Cette analyse montre l'homogénéité de la forêt dans cette région et l'existence d'un segment toposéquentiel de végétation. Le principal facteur de variation est le cycle silvigénétique, consideré comme processus d'autorégénération par création et cicatrisation de chablis.
\end{abstract}

\section{INTRODUCTION}

Dans le cadre du projet "Exploração e Manejo da Floresta Tropical Úmida", il nous fut demandé d'apprécier l'homogénéité de la végétation couvrant les trois bassins hydro. graphiques situés au Sud de la route ZF-02. Pour ce faire, trois toposéquences étaient étudiées en même temps que fut réalisée une interprétation de la couverture photogramétrique. Les résultats ne montrent pas de différence entre les bassins, mais mettent en évidence une séquence structurale en rapport avec le modèle topographique. Par contre au stid-est des bassins, l'altituaie diminue très faibiement mais suffisamment pour modifier les forêts de plateau qui ne montrent pratiguement plus d'émergents.

A partir de ces résultats et d'observations complémentaires, nous proposons ici une analyse de la structure et du dynamisme d'une forêt dense humide d'Amazonie Centrale.
Selon le volume 18, folha 20 Manaus du projet "RADAM BRASIL", nos bassins se situent sur un relief "acidentádo, prevalescendo o dissecamento em cristas, revestido pela subformação da floresta densa com, árvores emergentes - Abrangendo uma área expressiva, está intercalada com a floresta aberta de palmeira, que ocupa os vales encaixados, entre cristas, formando uma comunidade" (p. 506)

Nos observations permettent de préciser ce schéma: la formation végétale dominante est une forêt dense tropicale de terre ferme ("floresta densa tropical de terra firme"). Sur les sols hydromorphes, généralement sableux: les palmiers dominants provoquent une ouver. ture plus importante de la couverture forestière ("floresta aberta" ou "cocal"). Enfin deux petites taches de "campinas abertas" appa. raissent dans la partie méridionale du bassin I.

\section{MATÉRIEL ET MÉTHODES}

Les trois toposéquences étudiées se situent au Km. 14 dans le bassin I, Km. 20 et $\mathrm{Km} .22$ dans le bassin III; une quatrième au $\mathrm{Km} .38$, hors des bassins, nous a servi à évaluer l'homogénéité de la région entre la BR 174 et le Cuieiras.

Sur chacuns de ces toposéquences nous décrivons les structures végétales, leurs proportions relatives et leur disposition dans l'espace, d'après une méthode déjá décrite (Guil. laumet \& Kahn, 1978 et 1979) et utilisée dans la description et l'analyse du dynamisme de la végétation en Côte d'lvoire.

Nous ne rappellerons ici que les objectifs et principes qui sont à la base de cette métho-

(1) - Botanistes de l'Office de la Recherche Scientifique et Technique Outre-Mer (ORSTOM) France - Departamento de Ecologia. Instituto Nacional de Pesquisas da Amazônia, Manaus.

Convênio CNPq/CNRS: Projeto “Estudo das Modificações Ecológicas ligado ao Manejo Agro-Silvicultural da Mata". 
de et ne présenterons que les éléments qui sont utilisés dans cette étude.

Son objectif est de permettre une description aussi exacte que possible de la réalité, qui peut ensuite être interprétée selon les besoins. Parmi les critères utilisés par les botanistes pour décrire la végétation, seuls les critères structuraux sont "intrinséques à la végétation, quantifiables et généralisables" (Descoings, 1976), ce sont ceux que nous avons utilisés.

II nous a fallu définir quelques concepts nouveaux correspondant à des réalités observables et établir une typologie capable d'intégrer l'ensemble des données. Pour nommer ces concepts, des néologismes ont été créés.

Nous considérons deux volumes caractéristiques :

- Le phytoplexion, volume structuralement homogène de végétation.

- L'hoplexol, volume d'organisation du phytoplexion, se définit comme un continuum spatial horizontal constitué des éléments végétaux et du milieu ambiant dont la nature et la disposition le différencient des volumes inférieur et supérieur. L'hoplexol recouvre plus ou moins les notions de strate, interstrate, "canopy, layer, story", etc.

L'analyse d'un phytoplexion se fait en décrivant les hoplexols qui le composent.

L'analyse des hoplexols, ou diagnose, consiste à en décrire et quantifier les éléments constitutifs.

Toute plante arborescente, ou mieux tout phanérophyte érigé ou lianescent, ligneux ou non, présente un système conducteur (axes aériens et racinaires) reliant deux systèmes assimilateurs (feuilles et racines absorbantes) séparés dans l'espace.

Nous considérons pour ces plantes deux entités structurales et fonctionnelles :

- les systèmes assimilateurs

- les systèmes conducteurs.

Si, chez la majorité des plantes de la forêt tropicale humide, ces deux entités existent, les systèmes conducteurs des plantes non ligneuses, de taille réduite, sont moins différenciés et ont une signification écologique différente par leur occupation et situation spatiales. Les zones de conduction et les zones d'assimilation sont intimement liées et nous considérons alors l'ensemble de la partie aérienne du végétal.

Une description de la végétation serait incomplète si elle ne tenait pas compte des plantules et jeunes plants et de la matière vé. gétale morte.

II est indispensable de bien noter que les éléments de diagnose décrivent des ensembles et qu'ils ne peuvent être appliqués à un individu ou à une partie d'individu végétal.

\section{Parties aÉRIEnNes}

ENSEMBLES DES PLANTES DONT LES SYSTÈMES ASSIMILATEURS ET CONDUCTEURS SONT SÉPARÉS DANS I'ESPACE

Systèmes assimilateurs (feuillages et axes associés): éléments de diagnose de la catégorie des - physe.

Paliphyse: Ensemble des feuillages des arbres présentant leur expansion maximale.

- Morphologie : ces feuillages ont généralement une forme plus large que haute, globuleuse, en coeur ou en tronc de cône renversé évasé vers le haut.

- Stratégie : ces feuillages appartiennent à des arbres ayant acquis leur expansion maximale.

Prophyse: Ensemble des feuillages des arbres en cours de croissance.

- Morphologie : ces feuillages ont une forme en fuseau, plus haute que large.

- Stratégie: une telle forme indique que l'arbre croît en hauteur, limitant son expansion latéiale. Ces arbres sont en train de réaliser leur modèle de croissance (Hallé \& Oldeman, 1970).

Monophyse. Ensemble des feuillages des arbres mono-et oligoblastes.

- Morphologie: les feuillages sont groupés en touffes au sommet des tiges. 
- Stratégie: la croissance des plantes qui constituent le monophyse est généralement lente et leur occupation de l'espace est limitée par leur mode de croissance (absence de ramification) mais se trouve accrue par les dimensions généralement importantes des feuillages. Ici, !es seuls feuillages appartenant au monophyse sont ceux des palmiers.

Pléiophyse: ensemble des feuillages des lianes. Dans les forêts étudiées, les lianes sont toujours présentes, mais peu nombreuses. Aussi le pléiophyse n'apparait-il pas dans les tableaux I à à IV.

Systèmes conducteurs : éléments de diagnose de la catégorie des - agé.

Stylagé : ensemble des troncs des Dicotylédones.

Dendrigé : ensemble des branches qui se distinguent, dans l'espace, des feuillages. II correspond soit aux principales vagues de réitérations (Oldeman, 1974), soit à des branches séquentieiles ou ramifications développées lorsq̣ue la plante ne réitère pas.

Stipiagé : $€$ nsemble des tiges ou stipes des Monocotylédones arborescentes, ici des palmiers.

Ophiagé : ensemble des tiges ou troncs des lianes, trop peu représentés dans les parcelles étudiées pour apparaitre dans les tableaux I à IV.

1.2. Ensembles des plantes dont les systèmes assimilateurs et conducteurs ne sont pas individualisés dans l'espace.

Néophytion: plantules et jeunes plants des formes arborescentes, et des lianes lig. neuse.

Gramer: ensemble des plantes herbacées graniniformes.

Kortode : ensemble des herbes ni graminiformes, ni prostrées (le phorophytion non utilisé ici). Ce sont des Dicotylédones, Monocotyléclones et Ptéridophytes, aux formes variées.

Gramen et kortode incluent respectivement leurs plantules, le plus souvent mêlées aux formes adultes et qui n'ont pas la signification. dynamique du néophytion.

\section{Parties Souterraines}

Les axes racinaires de conduction et d'assimilation forment des ensembles qui ont reçu des noms mais que nous ne citerons pas n'ayant pas été utilisés dans ce travail (cf. Guiliaumet \& Kahn, 1979).

\section{MATIÈ'RE VÉGÉTALE MORTE}

Nécrophytion: matière végétale morte non décomposée. La forme des organes végétaux est toujours reconnaissable.

\section{QUELQUES TERMES COMPOSÉS}

Le langage proposé permet de décrire tous les cas et nous définissons ci-dessous 3 termes composés qu'il a fallu utiliser pour les éléments de diagnoses intermédiaires.

Propaliphyse (de pro- et paliphyse): ensemble des feuillages des arbres qui ont presque atteint leur hauteur maximale mais dont l'expansion latérale n'est pas terminée.

Promonophyse (de pro- et monophyse) : ensemble des couronnes de palmiers qui n'ont pas atteint leur expansion maximale. Il est l'équivalent du terme prophyse.

Néomonopinyse (de néophytion et monophyse): ce terme qualifie l'ensemble des plan tules et très jeunes sujets de palmiers.

\section{LA QUANTIFICATION DES ÉLÉMENTS DE DIAGNOSE}

If s'agit diune quantification relative des éléments végétaux entre eux; l'importance relative de leurs volumes permet de distinguer 5 niveaux qui s'écriront sous la forme suivante :

1. Prophyse

2. Pro-Paliphyse

3. Paliphyse, prophyse

4. Paliphysa, phase prophyse

5. Paliphyse, stigme propliyse

Nous n'avons présenté dans ce travail (Tab. I à IV) que l'essentiel des diagnoses majeures des hoplexols, relatif à l'identification et la quantification des principaux éléments. 
TABLEAU I - Analyse morpho-structurale des toposéquences: Relevés de plateaux.

\begin{tabular}{|c|c|c|c|c|c|c|}
\hline & N.CH & $\mathrm{Km} \cdot 14$ & N. ${ }^{\circ} \mathrm{H}$ & Km. 20 & Km. 22 & Km. 38 \\
\hline 1 & $\begin{array}{l}18-35 \\
17-26 \\
16-22\end{array}$ & $\begin{array}{l}\text { Paliphysen } \\
\text { Propaliphysen } \\
\text { Paliphysen-1 }^{n}\end{array}$ & $\begin{array}{l}17-40 \\
16-30 \\
15-22\end{array}$ & $\begin{array}{l}\text { Paliphysen } \\
\text { Paliphysen-1,n } \\
\text { Palin-1, propaliphysen }\end{array}$ & $\begin{array}{l}\text { 16-25 Propalin-Paliphysen } \\
\text { 15-20 Propaliphysen, phase paliphysen }\end{array}$ & $\begin{array}{l}\text { 18-40 Paliphysen } \\
\text { 17-30 Paliphysen-1, phase dendrigé } \\
\text { 16-23 Paliphysen-2, prophyse }\end{array}$ \\
\hline 2 & $\begin{array}{l}15-16 \\
14-13 \\
13-11\end{array}$ & $\begin{array}{l}\text { Prophyse, phase paliphyse } \\
\text { Prophyse } \\
\text { Prophyse }\end{array}$ & $\begin{array}{l}14-17 \\
13-13\end{array}$ & $\begin{array}{l}\text { Pali-Prophyse } \\
\text { Prophyse }\end{array}$ & 14-14 Prophyse & $\begin{array}{l}\text { 15-17 Prophyse, propaliphyse } \\
14-12 \text { Styla-Prophyse }\end{array}$ \\
\hline 3 & $\begin{array}{l}12-8 \\
11-6 \\
10-4,5 \\
9-3,5 \\
8-2,2\end{array}$ & $\begin{array}{l}\text { Styla-Prophyse } \\
\text { Styla-Prophyse } \\
\text { Pro-Stylagé } \\
\text { Pro-Stylagé, monophyse } \\
\text { Pro-Stylagé, monophyse }\end{array}$ & $\begin{array}{c}12-9 \\
11-6 \\
10-4 \\
9-3 \\
8-2,2\end{array}$ & $\begin{array}{l}\text { Styla-Prophyse, phase monophy- } \\
\text { se } \\
\text { Pro-Stylagé, phase monophyse } \\
\text { Pro-Stylagé, phase monophyse } \\
\text { Stylagé-Pro-Monophyse } \\
\text { Mono-Pro-Stylagé }\end{array}$ & \begin{tabular}{|ll}
$13-8$ & Prophyse \\
$12-6$ & Styla-Prophyse \\
$11-4,5$ & Prophyse-Stylagé \\
$10-3$ & Styla-Pro-Monophyse \\
$9-2$ & Styla-Monophyse, prophyse \\
$8-1,6$ & Pro-Stylagé
\end{tabular} & $\begin{array}{ll}13-8 & \text { Styla-Prophyse } \\
12-5,5 & \text { Pro-Stylagé, phase monophyse } \\
11-4 & \text { Pro-Stylagé } \\
10-3 & \text { Pro-Mono-Stylagé } \\
9-2,2 & \text { Pro-Mono-Stylagé } \\
8-1,5 & \text { Pro-Stylagé }\end{array}$ \\
\hline 4 & $\begin{array}{l}7-1,4 \\
6-1 \\
5-0,6 \\
4-0,3 \\
3-0,2 \\
2-0,08 \\
1-0,04\end{array}$ & $\begin{array}{l}\text { Pro-Stylagé, monophyse } \\
\text { Pro-Stylagé } \\
\text { Pro-Stylagé } \\
\text { Néo-Stylagé, phase kortode } \\
\text { Néo-Stylagé, phase kortode } \\
\text { Stylagé, phase néophytion } \\
4 \text { Nécro-Stylagé }\end{array}$ & $\begin{array}{l}7-1,6 \\
6-1,2 \\
5-0,8 \\
4-0,5 \\
3-0,3 \\
2-0,2 \\
1-0,03\end{array}$ & $\begin{array}{l}\text { Stylagé, prophyse, phase mono- } \\
\text { physe } \\
\text { Pro-Stylagé, phase monophyse } \\
\text { Pro-Stylagé, phase kortode, pha- } \\
\text { se gramen } \\
\text { Néo-Stylagé, kortode } \\
\text { Néo-Stylagé } \\
\text { Néo-Stylagé } \\
\text { Nécro-Stylagé }\end{array}$ & $\begin{array}{l}\text { 7-1,1 Pro-Stylagé } \\
\text { 6-0,8 Pro-Stylagé } \\
\text { 5-0,5 Néophytion-Stylagé } \\
\text { 4-0,35 Néophytion-Stylagé } \\
\text { 3-0,2 Néo-Stylagé } \\
\text { 2-0,08 Nécro-Stylagé, phase néophytion } \\
\text { 1-0,03 Nécro-Stylagé }\end{array}$ & $\begin{array}{l}\text { 7-0-9 Pro-Stylagé } \\
\text { 6-0,6 Pro-Stylagé } \\
5-0,4 \text { Néophytion-Stylagé } \\
\text { 4-0,25 Néo-Stylagé } \\
\text { 3-0,15 Néo-Stylagé } \\
\text { 2-0,07 Néo-Stylagé } \\
\text { 1-0,03 Nécro-Stylagé }\end{array}$ \\
\hline
\end{tabular}

1. essembles arborescents supérieurs; 2. ensemb'es arborescents moyens; 3 . ensembles inférieurs arbustifs; 4. ensembles inférieurs arbustifs et herbacés, Pour chaque toposéquence le ler chitfre indique le número de l'hoplexol, le second la hauteur supérieure en mètres de celui-ci. 
LES ENSEMBLES FORESTIERS

Après avoir identifié et quantifié les différents hcplexols de chaque phytoplexion observé, hous les avons regroupes en 4 niveaux: arborescent supérieur, arborescent moyen, arbustif, arbustif inférieur et herbacé.

Nous avons largement utilisé la notion d'ensembles d'arbres forestiers introduite par Oldeman en 1974 :

- ensemble d'avenir : arbres et arbustes en cours de croissance et conformes à leur modele initial.

- ensemble du présent: arbres ayant atteint leur pleine maturité et qui tendent à réaliser leur biomasse maximale.

Nous avons été amenés à distinguer un ensemble du présent réalisé d'un ensemblo en cours de réalisation. Les feuillages du premier sont décrits en tant que paliphyse, ceux du second comme propaliphyse.

Les arbres de l'ensemble du présent s'épanouissant à une même hauteur constituent un "ensemble structural" défini par Oldeman (1974).

\section{ASPECTS PRATIQUES}

Un relevé morpho-structural consiste à observer et à décrire les hoplexols, successive. ment et de bas en haut.

\section{RESULTATS ET DISCUSSIONS}

COMPARAISON DES TOPOSĖQUENCES DES KM. 14 ET 20.

LES NIVEAUX ARBORESCENTS SUPÉRIEURS.

Plateaux (Tab. 1.1. et Fig. 1.1. et 2.1.)

Les relevés de plateau mettent en évidence 3 hoplexols constitués de paliphyse et pro. paliphyse, éléments qui traduisent la présencc d'ensembles du présent réalisés et en cours de réalisation.

Au Km. 14, les couronnes d'un premier ensemble struciural réalisé (paliphysen]), entre 26 et $35 \mathrm{~m}$, dominent les feuillages d'un ensemble en cours de réalisation (propaliphy- sen), entre 22 et $26 \mathrm{~m}$, destitiés à s'associer aux premiers, et d'un second ensemble structural (paliphysen-1), entre 16 et $22 \mathrm{~m}$.

$\mathrm{Au} \mathrm{Km} .20$, les feuillages du promier en. semble structural (paliphysen), s'étendent sur les 2 hoplexols supérieurs (16 et 17) et s'associent dans l'hoplexol 16, entre 22 et $30 \mathrm{~m}$. au sommet des couronnes d'un second ensemble structural (paliphysen-1) qui lui-même, dans sa partie basse, hoplexol 15, entre 17 et $22 \mathrm{~m}$, se mélange aux feuillages d'un ensemble en cours de réalisation (propaliphysen).

Hauts de pente (Tab. II. 1. et Fig. 1.2. et 2.2.)

Les hauts de pente sont caractérisés par deux hoplexols (15 et 16) essentiellement constitués de paliphyse, correspondant aux feuillages supérieurs de deux ensembles structuraux.

Au Km. 14, apparaissent dans l'hoplexol inférieur, entre 18 et $24 \mathrm{~m}$, des feuillages d'arbres non entièrement réalisés (propaliphyse) destinés à atteindre l'hoplexol supérieur.

Pentes (Tab. III. et Fig. 1.3. et 2.3.)

Dans cette situation topographique, les niveaux arborescents supérieurs sont caractérisés par deux hoplexols. Le même paliphysen se trouve dans les 2 hoplexols, signifiant que les teuillages d'un ensemble du présent s'étalent largement entre 18 et $24 \mathrm{~m}$ au Km. 14, entre 15 et $28 \mathrm{~m}$ au $\mathrm{Km} .20$.

Sur la toposéquence du Km. 14, l'hoplexol 14 , entre 18 et $24 \mathrm{~m}$, montre un propaliphyse qui indique la présence d'un ensemble structurai en cours de réalisation. Au Km. 20, c'est un ensemble d'arbres d'avenir qui apparait avec le prophyse de l'hoplexol 12, entre 15 et $22 \mathrm{~m}$, associé à la base des couronnes (paiiphysen) et aux grosses branches (dendrigé) de l'ensemble structurá⿴囗 le plus élevé.

Bas-londs (Tab. IV. 1 et Fig. 1.4. et 2.4.)

La structure du niveau arborescent supérieur se complique quelque peu dans les bas-fond's. II y a toujours 2 hoplexols, mais apparition d'un nouvel élément de diagnose. monophyse, qui traduit la présence des touffes de feuilles de palmiers. 
TABLEAU II - Analyse morpho-structurale des toposéquences Relevés de hauts de pente.

\begin{tabular}{|c|c|c|c|c|c|c|c|c|}
\hline \multicolumn{3}{|c|}{$\mathrm{N} \cdot{ }^{\circ} \mathrm{H} \quad \mathrm{m}$} & \multicolumn{2}{|c|}{$\begin{array}{ll}\mathrm{N} . \mathrm{H} & \mathrm{Km} .20\end{array}$} & \multicolumn{2}{|c|}{ N.PH m $\quad$ Km. 22} & \multicolumn{2}{|r|}{ Km. 38} \\
\hline 1 & $\begin{array}{l}16-35 \\
15-24\end{array}$ & $\begin{array}{l}\text { Paliphysen }^{n} \\
\text { Paliphysen-1, propaliphysen }^{n}\end{array}$ & $\begin{array}{ll}16-30 \\
15-22\end{array}$ & $\begin{array}{l}\text { Paliphysen } \\
\text { Paliphysen-1 }^{-1}\end{array}$ & $\begin{array}{l}16-30 \\
15-22\end{array}$ & $\begin{array}{l}\text { Propaliphysen } \\
\text { Propaliphysen-1, paliphysen }^{n}\end{array}$ & $\begin{array}{l}18-30 \\
17-22\end{array}$ & $\begin{array}{l}\text { Paliphysen } \\
\text { Paliphysen,n-1, prophyse }^{\text {nal }}\end{array}$ \\
\hline 2 & $\begin{array}{l}14-18 \\
13-12\end{array}$ & $\begin{array}{l}\text { Propaliphyse } \\
\text { Prophyse }\end{array}$ & 14-15 & $\begin{array}{l}\text { Prophyse, phase paliphyse, pha- } \\
\text { se monophyse } \\
\text { Prophyse }\end{array}$ & $\begin{array}{l}14-16 \\
13-12\end{array}$ & $\begin{array}{l}\text { Prophyse, paliphyse } \\
\text { Prophyse, stylagé }\end{array}$ & $\begin{array}{l}16-16 \\
15-12\end{array}$ & $\begin{array}{l}\text { Pro-Propaliphyse } \\
\text { Styla-Prophyse }\end{array}$ \\
\hline 3 & $\begin{array}{l}12-9 \\
11-6 \\
10-4,5 \\
9-3 \\
8-2,5\end{array}$ & $\begin{array}{l}\text { Styla-Mono-Prophyse } \\
\text { Styla-Prophyse } \\
\text { Styla-Prophyse } \\
\text { Pro-Stylagé, monophyse } \\
\text { Styla-Pro-Monophyse }\end{array}$ & $\begin{array}{l}12-10 \\
11-7 \\
10-5 \\
9-3 \\
8-2,5\end{array}$ & $\begin{array}{l}\text { Styla-Pali-Prophyse } \\
\text { Stylagé, phase prophyse } \\
\text { Pro-Stylagé, phase monophyse } \\
\text { Stylagé-Monophyse } \\
\text { Stylagé-Monophyse }\end{array}$ & $\begin{array}{l}12-8 \\
11-5,5 \\
10-4 \\
9-3 \\
8-2\end{array}$ & $\begin{array}{l}\text { Styla-Prophyse } \\
\text { Stylagé-Prophyse, monophyse } \\
\text { Styla-Prophyse, monophyse } \\
\text { Pro-Mono-Stylagé } \\
\text { Pro-Mono-Stylagé }\end{array}$ & $\begin{array}{c}14-9 \\
13-6 \\
12-4,5 \\
11-3 \\
10,2,5 \\
9-2\end{array}$ & $\begin{array}{l}\text { Styla-Prophyse } \\
\text { Pro-Stylagé, phase monophyse } \\
\text { Pro-Stylagé, phase paliphyse, } \\
\text { phase monophyse } \\
\text { Pro-Monophyse, stylagé } \\
\text { Prophyse, stylagé, monophyse } \\
\text { Promono-Prophyse, stylagé }\end{array}$ \\
\hline 4 & $\begin{array}{l}7-1,8 \\
6-1 \\
5-0,7 \\
4-0,4 \\
3-0,2 \\
2-0,1 \\
1-0,03\end{array}$ & $\begin{array}{l}\text { Styla-Pro-Monophyse } \\
\text { Pro-Stylagé, phase monophyse } \\
\text { Pro-Stylagé, phase kortode } \\
\text { Korto-Néophytion, stylagé } \\
\text { Néo-Stylagé, phase kortode } \\
\text { Stylagé, néophytion } \\
\text { Stylagé, nécrophytion }\end{array}$ & $\begin{array}{l}7-1,8 \\
6-1,3 \\
5-0,9 \\
4-0,6 \\
3-0,3 \\
2-0,2 \\
1-0,03\end{array}$ & $\begin{array}{l}\text { Pro-Stylagé, monophyse } \\
\text { Promono-Pro-Stylagé } \\
\text { Pro-Stylagé, promonophyse } \\
\text { Pro-Stylagé, promonophyse } \\
\text { Néo-Stylagé, phase kortode } \\
\text { Néo-Stylagé, phase kortode } \\
\text { Nécro-Stylagé }\end{array}$ & $\begin{array}{l}7-1,5 \\
6-1 \\
5-0,6 \\
4-0,45 \\
3-0,3 \\
2-0-15 \\
1-0,04\end{array}$ & $\begin{array}{l}\text { Pro-Stylagé, monophyse } \\
\text { Promono-Pro-Stylagé } \\
\text { Pro-Stylagé, promonophyse } \\
\text { Néo-Stylagé } \\
\text { Néo-Stylagé } \\
\text { Néo-Stylagé } \\
\text { Nécro-Stylagé }\end{array}$ & $\begin{array}{l}8-1,5 \\
7-1,1 \\
6-0,8 \\
5-0,5 \\
4-0,35 \\
3-0,2 \\
2-0,1 \\
1-0,03\end{array}$ & $\begin{array}{l}\text { Promono-Prophyse, stylagé } \\
\text { Promono-Pro-Stylagé } \\
\text { Pro-Stylagé } \\
\text { Néophytion-Stylagé } \\
\text { Néophytion-Stylagé } \\
\text { Néo-Stylagé } \\
\text { Néo-Stylagé } \\
\text { Nécro-Stylagé }\end{array}$ \\
\hline
\end{tabular}

1. ensembles arborescents supérieurs; 2 ensembles arborescents moyens; 3 ensembles inférieurs arbustifs et herbacés.

Pour chaque toposéquence le ler chifre indique le numéro de l'hoplexol, le second la hauteur supérieure en mètres de celui-ci. 
L'hoplexol supérieur, 14 entre 20 et $25 \mathrm{~m}$, au $\mathrm{Km}$. 14, comporte un paliphyse et un propaliphyse, feuiliages de deux ensembles structuraux, l'un réalisé, l'autre en cours de réalisation, dont ies bases se retrouvent dans I'hoplexol sous-jacent, entre 15 et $20 \mathrm{~m}$, associées à des couronnes de palmiers (monophyse) et aux grosses branches des plus grands arbres (dendrigé). Le palmier. Jessenia bataua, participe lui-même à la voûte.

$\mathrm{Au} \mathrm{Km} .20$, deux paliphyses se superposent, le premier entre 24 et $30 \mathrm{~m}$, le second entre 16 et $24 \mathrm{~m}$, indiquant la présence de 2 ensem. bles structuraux, associés l'un et l'autre à un monophyse. Le monophyse supérieur est constitué des feuillages de Mauritia flexuosa et Jessenia bataua, le second de Mauritia aculeata et Euterpe sp.

L'analyse des niveaux supérieurs montre que, dans une même situation topographique, la structure des hoplexols est semblable, les niveaux arborescents supérieurs des deux toposéquences sont donc comparables.

Les variations sont dues au développement des arbres, ce qui se traduit par les différentes diagnoses, paliphyse, propaliphyse, prophyse, correspondant à des ensembles d'arbres du présent réalisés ou en cours de réalisation et à des ensembles d'avenir.

LES NIVEAUX ARBORESCENTS MOYENS (Tab. I. 2. II 2, III.2. IV.2 et Fig. 1 et 2.)

Dans tous les relevés, il apparait I hoplexol dont les éléments paliphyse, monophyse ou propaliphyse mettent en éviderice la présence d'un ensemble d'arbres du présent réalisé ou en cours de réalisation.

Le nombre d'hoplexols varie de 1 à 3 sur les plateaux, 1 à 2 sur les pentes et bas-fonds; il est de 2 sur les hauts de pente. Ces variations sont en partie liées à l'importance des ensembles d'avenir.

II faut signaler que, dans tous les relevés, l'espace est occupé entièrement par des feuillages et que la variation du nombre d'hoplexols ne se traduit par aucun changement profond de structure. Ainsi sur la pente de la toposéquence du: $\mathrm{Km} .20$, il n'y a qu'un ensem- ble d'arbres, et les feuillages occupent le même espace aue 2 ou 3 ensembles dans les autres positions.

Dans les zones basses hydromorphes, ces niveaux sont assentiellement constitués d'arbres jeunes et de palmiers. Au Km. 20, nous notons la présence de la base des feuillages (paliphysen-1) des arbres du présent des niveaux supérieurs.

\section{LES NIVEAUX INFÉRIEURS}

1.3.1. Les ensembles d'arbustes (Tab. I 3, II. 3, III. 3. et IV. 3) .

Sur le plateau et le haut de pente, d'une toposéquence à l'autre, les hoplexols sont respectivement au nombre de 5 et varient de 4 à 5 dans la pente; le bas-fond du Km. 14 en donne 4 et celui du Km. 20, 3 seulement.

Ces niveaux sont caractérisés par la présence d'éléments du monophyse, feuillages de palmiers qui jouent un rôle très important dans la physionomie et la structure. Ce sont Astrocaryum aft. javarense et Attalea attaleoides constituant une couverture végétale dense entre 1,8 et $2,5 \mathrm{~m}$ qui va en diminuant jusque vers $4 \mathrm{~m}$.

Mais sur le relevé de haut de pente de la toposéquence du $\mathrm{Km} .14$, Oenocarpus minor et Astrocaryum munbaca sont abondants entre 6 et $9 \mathrm{~m}$, alors que la même position au $\mathrm{Km} .20$ est marquée par la présence d'un paliphyse (hoplexol 12 entre 7 et $10 \mathrm{~m}$ ) marquant les feuillages d'un ensemble d'arbustes du pré. sent.

Dans les bas-fonds, tous les hoplexols sont caractérisés par le monophyse: les palmiers apparaissent à tous les niveaux, ce sont des adultes de Manicaria saccifera, Attalea aff. spectabilis et Astrocaryum acaule ou des jeunes Jessenia bataua, Mauritia flexuosa et Euterpe sp (promonophyse). Le prophyse, souvent répété dans les diagnoses, représente l'ensemble d'arbres d'avenir.

1.32 . Les niveaux les plus bas: arbustes et herbes (Tab. I. 4, II. 4, III. 4 et IV. 4.).

Les 3 premières positions donnent, sur les 2 toposéquences, le même nombre d'hoplexols, 
TABLEAU III - Analyse morpho-structurale des toposéquences: Relevés de pentes.

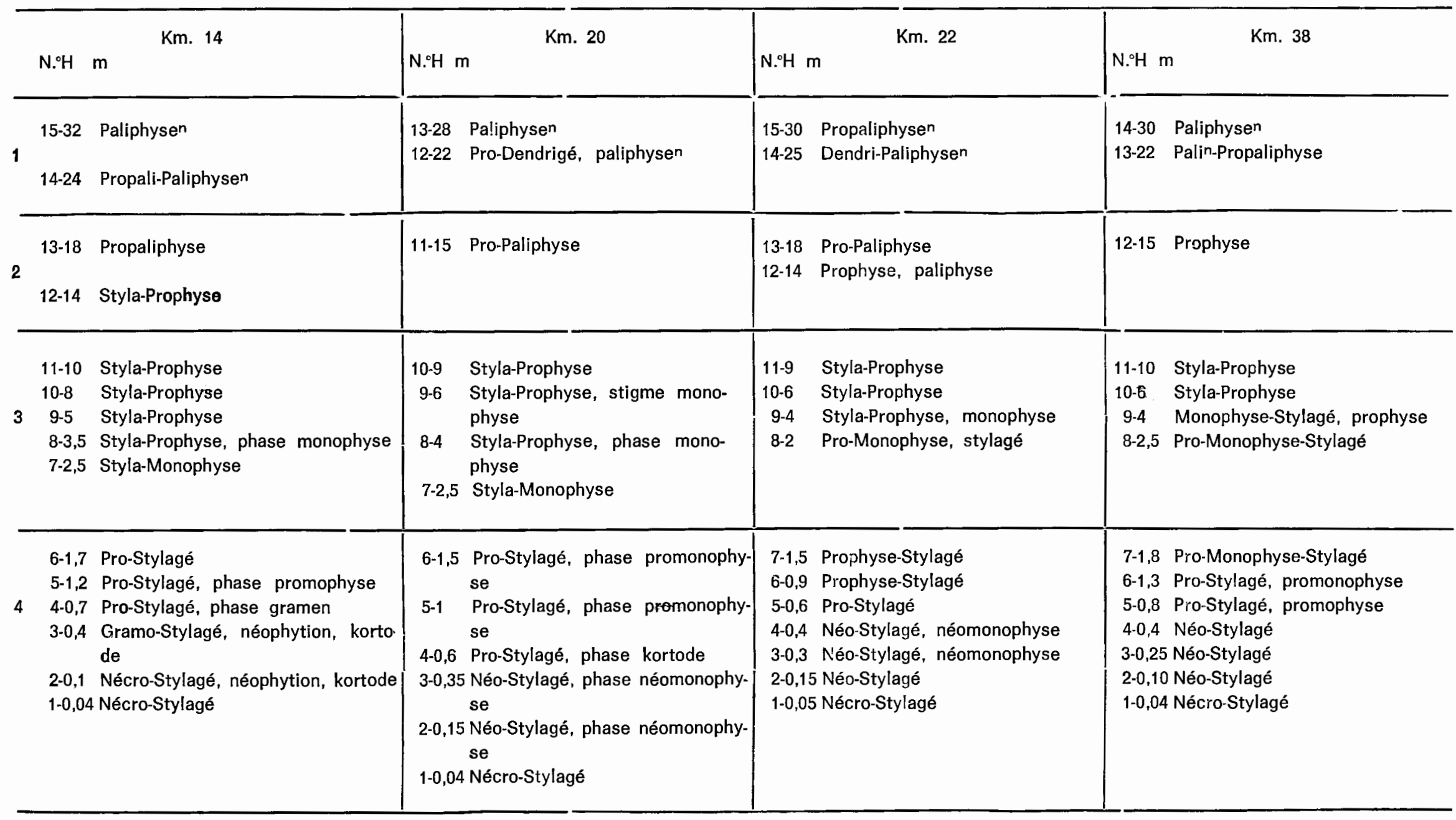

1. ensembles arborescents supérieurs; 2. ensembles arbores cents moyens; 3 . ensembles inférieurs orbustifs et herbocés. Pour chaque toposéquence le ler chiffre incique le numéro de l'hoplexol, le second la hauteur supérieure en mètres de celui-ci. 

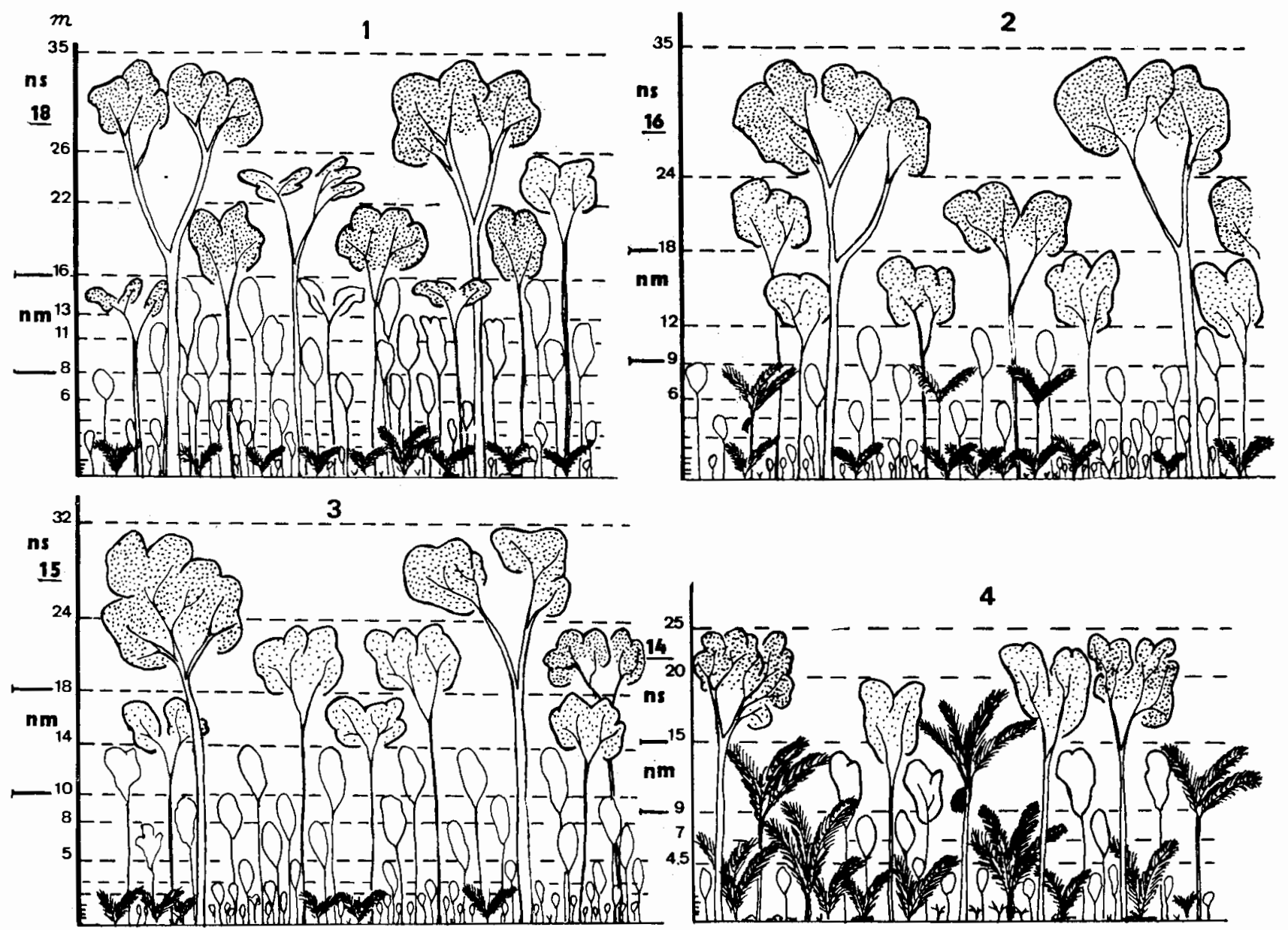

Fig. 1 - lllustrations de la végétation sur la toposéquence du $\mathrm{Km} .14$, bassin I. 1. plateau; 2 . haut de pente; 3 . pente; 4 . bas-fond. ns. niveau arborescent supérieur; $\mathrm{nm}$. niveau arborescent moyen. Les hoplexols sont matérialisés par des lignes discontinues, et leurs hauteurs en mètres données à gauche de chaque schéma. Le chiffre soulingé indique le rang de l'hoplexol supérieur, ou le nombre total d'hoplexols. Les couronnes d'arbres apparaissent en pointillé dense quand elles appartiennent au paliphyse, en pointillé espacé quand elles appartiennent au propaliphyse, en blanc dans le cas du prophyse.

soit 7,7 et 6 . Au $\mathrm{Km} .14$, le bas-fond en a 7 , pour 6 seulement au $\mathrm{Km} .20$.

Les différences portent uniquement sur la répartition verticale et le coricenu des hoplexols. Sur le plateau entre 0 et $0,3 \mathrm{~m}$, il y a 4 hoplexois au Km. 14, 3 seulement au Km. 20 où les plantules sont moins abondantes. Sur la pente, l'hoplexol 4, au Km. 14, est marqué par la présence d'une phase gramen, alors qu'au $\mathrm{Km}$. 20, les hoplexols 2 et 3, par uns phase néomonophyse correspondant à un en. semble de plantules de palmiers.

En bas-fonds, ces niveaux sont caractérisés par de nombreuses plantules de palmiers (néomonophyse), de plantes herbacées (kortode et gramen) telles des espèces de Rapatéacées.
RÉSUMÉ

\section{Comparaison entre les deux toposéquences}

Sur une même position topographique, on peut dégager les caractéristiquies structurales suivantes :

- Ensembles arborescents supérieurs. parraite similitude d'une toposéquence à l'autre; il y a 2 ou 3 hoplexols (plateaux) mettant en évidence la présence de deux ensembles structuraux ( 1 seul sur la pente du Km. 20) et piesque toujours d'un ensemble en cours de réalisation ou d'avenir. Les taibles différences sont dues à la dynamique interne de la forêt. 
TABLEAU IV - Analyse morpho-structurale des toposéquences: Relevés de bas-fonds.

\begin{tabular}{|c|c|c|c|c|c|c|c|c|}
\hline & N.'H $\mathrm{m}$ & Km. 14 & N.'H m & Km. 20 & N.PH m & Km. 22 & N.'H m & $\mathrm{Km} .38$ \\
\hline 1 & $13-20$ & $\begin{array}{l}\text { Propalin-Paliphysen } \\
\text { Dendri-Propaliphysen-1, monophyse } \\
\text { phase paliphysen }\end{array}$ & $\begin{array}{ll}13-30 & P \\
12-24 & P\end{array}$ & $\begin{array}{l}\text { Paliphysen, monophyse } \\
\text { Paliphysen-1, phase monophyse }^{n-1}\end{array}$ & $\begin{array}{ll}15-30 & \mathrm{~Pa} \\
14-22 & \mathrm{D}\end{array}$ & $\begin{array}{l}\text { Paliphysen, phase propaliphyse } \\
\text { Dendri-Paliphysen-1,n }\end{array}$ & $14-25 \mathrm{P}$ & Paliphyse, monophyse \\
\hline 2 & $12-15$ & Mono-Prophyse & $\begin{array}{ll}11-16 \\
10-12\end{array}$ & $\begin{array}{l}\text { Dendri-Monophyse, paliphysen-1 } \\
\text { Monophyse }\end{array}$ & $\begin{array}{ll}13-16 & \mathrm{Pr} \\
12-13 & \mathrm{Pr}\end{array}$ & $\begin{array}{l}\text { Propaliphyse, monophyse } \\
\text { Pro-Monophyse }\end{array}$ & $\begin{array}{ll}13-18 \\
12-12\end{array}$ & $\begin{array}{l}\text { Pro-Paliphyse, monophyse } \\
\text { Monophyse, propaliphyse }\end{array}$ \\
\hline 3 & $\begin{array}{l}11-9 \\
10-7 \\
9-4,5 \\
8-3\end{array}$ & $\begin{array}{l}\text { Mono-Prophyse } \\
\text { ES-Monophyse } \\
\text { ES-Promonophyse } \\
\text { Pro-Mono-ES }\end{array}$ & $\begin{array}{ll}9-8 & F \\
8-5 & E \\
7-3 & E\end{array}$ & $\begin{array}{l}\text { Pro-ES, monophyse } \\
\text { ES-Prophyse, promonophyse } \\
\text { ES-Promonophyse }\end{array}$ & $\begin{array}{rr}11-7 & \mathrm{E} \\
10-5 & \mathrm{PI} \\
9-3 & \mathrm{Pr}\end{array}$ & $\begin{array}{l}\text { ES-Monophyse } \\
\text { Pro-Monophyse, ES } \\
\text { Pro-Monophyse, ES }\end{array}$ & $\mid \begin{array}{ll}11-6 & \mathrm{~F} \\
10-4,5 & \mathrm{~F} \\
9-3 & \mathrm{~F} \\
8-2 & \mathrm{~F}\end{array}$ & $\begin{array}{l}\text { ES-Promonophyse, prophyse } \\
\text { Promono-Prophyse, ES } \\
\text { Promono-Prophyse, ES } \\
\text { Pro-Promonophyse, ES }\end{array}$ \\
\hline 4 & $\begin{array}{l}7-2 \\
6-1,1 \\
5-0,7 \\
4-0,4 \\
3-0,2 \\
2-0,1 \\
1-0,05\end{array}$ & $\begin{array}{l}\text { Promono-ES, prophyse } \\
\text { Promono-ES, prophyse } \\
\text { Promono-ES } \\
\text { Néomono-ES, kortode } \\
\text { Néomono-Néo-ES } \\
\text { Néo-ES } \\
5 \text { Nécro-ES }\end{array}$ & $\begin{array}{ll}6-1,8 & \\
5-1 & \\
4-0,6 & \\
3-0,3 & 1 \\
2-0,15 & 1 \\
1-0,03\end{array}$ & $\begin{array}{l}\text { ES-Promonophyse } \\
\text { Promono-ES } \\
\text { Néomono-ES, gramen } \\
\text { Néomono-ES, nécrophytion } \\
\text { Néo-ES, néomonophyse } \\
\text { Nécro-ES }\end{array}$ & $\begin{array}{ll}8-2 & P r \\
7-1,5 & P \\
6-1 & P r \\
5-0,7 & N \\
4-0,4 & N \\
3-0,2 & N \\
2-0,12 & N \\
1-0,05 & N\end{array}$ & $\begin{array}{l}\text { Pro-Monophyse, ES } \\
\text { Pro-ES } \\
\text { Pro-ES } \\
\text { Néomono-Gramen, ES } \\
\text { Néomono-Gramen, ES } \\
\text { Néomono-Néophytion, ES } \\
\text { Néomono-Néophytion, ES } \\
\text { Nécro-ES }\end{array}$ & $\begin{array}{l}7-1,3 \\
6-0,9 \\
5-0,6 \\
4-0,35 \\
3-0,2 \\
2-0,1 \\
1-0,02\end{array}$ & $\begin{array}{l}\text { Pro-Promonophyse, ES } \\
\text { Pro-Promonophyse, ES } \\
\text { Korto-Gramen, ES } \\
\text { Gramen-Néophytion, ES } \\
\text { Gramen-Néophytion, ES } \\
\text { Néo-ES } \\
\text { Nécro-ES }\end{array}$ \\
\hline
\end{tabular}

1. ensembles arborescents supérieurs; 2. ensembles arborescents moyens; 3 . ensembles inférieurs arbustifs et herbacés.

Pour chaque toposéquence le ler chiffre indique le numero de l'hoplexol, le second lo hauteur em mètres de celui.ci.

$\mathrm{ES}=$ Stipiagé + Stylagé 
- Ensembles moyens.

variations du nombre d'hoplexols. mais présence d'un ensemble d'arbres du présent achevé ou en cours de réalisation dans les 6 relevés.

\section{- Ensembles inférieurs.}

les relevés montrent une grande homogénéité structurale. La séquence du $\mathrm{Km}$. 20 est caractérisée par une répartition verticale des palmiers plus importante.

Variations sur la toposéquence (Tab. V).

Sur les deux toposéquences, en position de plateau le niveau arborescent supérieur est caractérisé par 3 hoplexols composés des feuillages diversement disposés de 3 ensembles structuraux réalisés ou en cours de réali-. sation, alors que sur les hauts de pente et les pentes il n'en reste plus que 2 .

Le cycle silvigénétique n'a pas, ici, atteint son stade ultime.

Dans les autres positions topographiques il n'y a que 2 ensembles arborescents audessus de $15 \mathrm{~m}$, quelquefois 1 seul et, en l'absence d'émergent, la voûte de la forêt apparait plus continue.

II n'y a pas de différence nette dans la structure de la forêt dans et hors des bassins, aux différences dues au cycle silvigénétique près.

Le niveau arborescent moyen semble le plus variable, te nombre d'hoplexols varie de 1 à 3 , indépendamment de la topographie (Tab. V). Notons cependant que l'espace est toujcurs occupé par des masses de feuillages
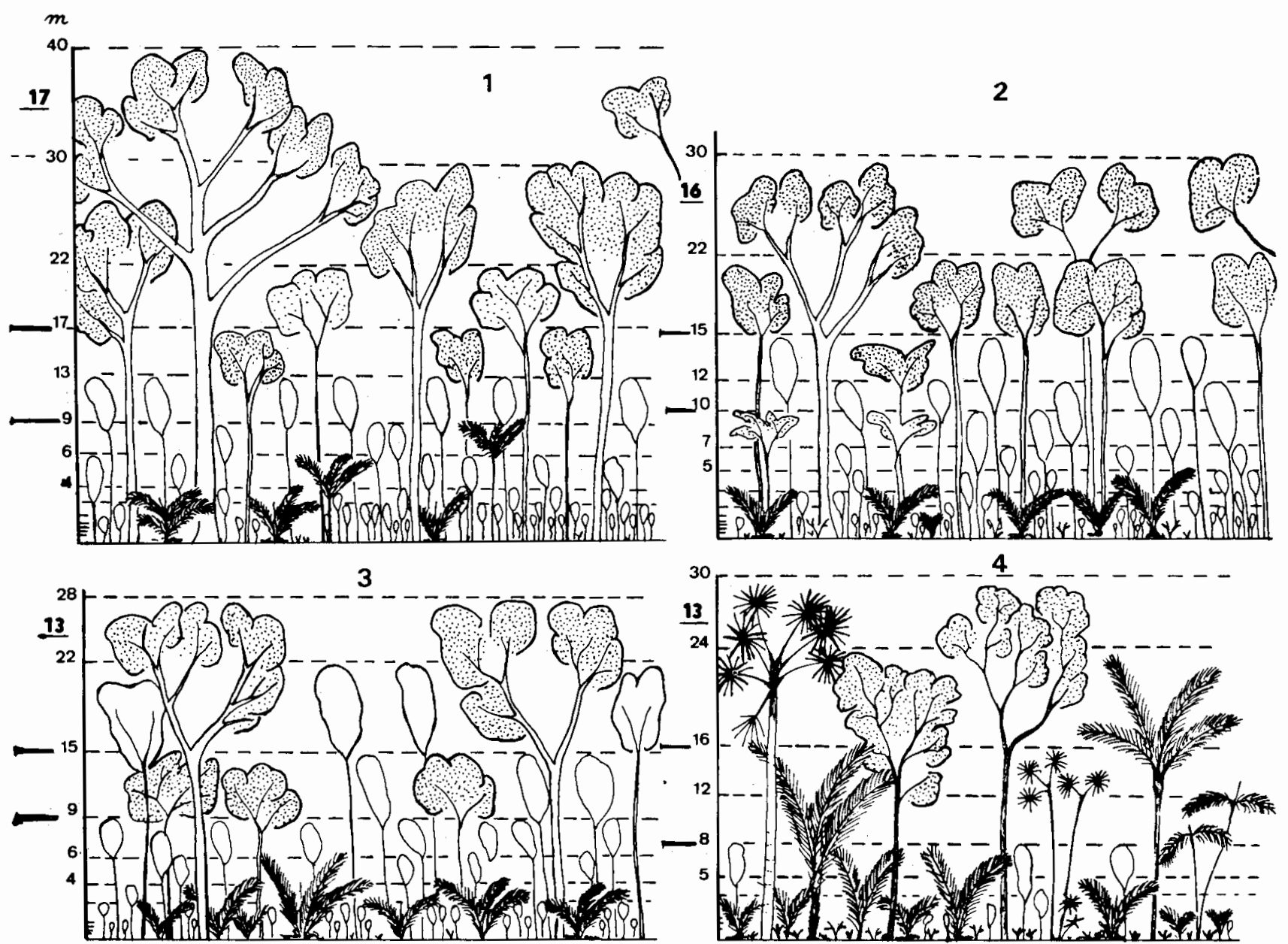

Fig. 2 - Illustrations de la végétation sur la toposéquence du $\mathrm{Km} .20$, bassin III. 1. plateau; 2, haut de pente; 3. pente; 4 . bas-fond. ns. niveau arborescent supérieur; nm. niveau arborescent moven. 
(éléments de diagnose en-physe), qu'il constitue un niveau trophique et que les variations structurales ne doivent pas entrainer de conséquences sensibles sur les gradients microclimatiques tels que l'interception de la pluie et de: l'énergie lumineuse.

Les différences structurales observées dépendent directement du cycle silvigénétique ou dynamisme interne de la forêt.

De nombreux auteurs (Richards, 1952; Cousens, 1965: Oldeman, 1974; Hallé et al. 1978; Hartshorn, 1978 et 1980, Denslow, 1980) ont montré le rôle des clairières naturelles ou cha'olis dans l'autorégénération de la forêt. Ces clairières naturelles sont créées par la chute d'un ou plusieurs arbres. Elles peuvent être de dimensions variées, sans toutefols excéder la surface couverte au sol par les cimes de deux grands arbres. II est clair que, dans une forêt, les arbres n'ont pas atteint le même stade de vieillissement et que les chablis se succèdent dans le temps et l'espace. Si bien que, à un moment donné, une forêt se présente comme une mosaïque de clairières naturelles à différents degrés de cicatrisation (Whitmore, 1978) .

Les effets de cette dynamique peuvent se résumer ainsi :

- le nombre d'arbres d'avenir dépend de l'état de régénération des chablis. Le nombre des hoplexols observés aux niveaux moyens et inférieurs sera d'autant plus élévé que l'ensemble d'avenir sera plus abondant.

Aux niveaux moyens, les variations sont indépendantes de la topographie. Nous notons la présence d'un hoplexol à paliphyse entre 9 et $15 \mathrm{~m}$ sur la pente de la toposéquence du $\mathrm{Km} .20$ qui peut s'expliquer par l'absence d'un ensemble structural au niveau supérieur.

La structure des niveaux inférieurs se réduit quelque peu sur les pentes avec 10 hoplexols au lieu de 12.

Les bas-fonds sont caractérisés par la dominance des palmiers tant aux niveaux supérieurs qu'au niveaux moyens et inférieurs.

La hauteur cle la forêt ne dépasse pas $35 \mathrm{~m}$, sauf sur le plateau de la toposéquence du $\mathrm{Km}$. 20 , ou un "angelim pedra" monte jusqu' à $40 \mathrm{~m}$.

Les plus faibles hauteurs s'observent sur les pentes.

LES TOPOSÉQUENCES DES KM. 22 ET 38.

La première toposéquence est située dans le bassin III, l'autre au-de là du bassin III. Leur étude étaiı destinée à confirmer ou infirmer les résultats obtenus sur les toposéquences des $\mathrm{Km} .14$ et 20 et à évaluer l'homogénéité structurale de cette région.

Les tableaux I à IV montrent la remarquable similitude d'un relevé à l'autre.

TABLEAU V - Nombre d'hoplexois par toposéquence et par niveau.

\begin{tabular}{|c|c|c|c|c|c|c|c|c|c|c|c|c|c|c|c|c|}
\hline & \multicolumn{4}{|c|}{$\mathrm{Km} \cdot 14$} & \multicolumn{4}{|c|}{ Km. 20} & \multicolumn{4}{|c|}{ Km. 22} & \multicolumn{4}{|c|}{$\mathrm{Km} .38$} \\
\hline & A & $B$ & $\mathrm{C}$ & D & $A$ & B & C & $D$ & $A$ & B & $\mathrm{C}$ & D & A & B & C & D \\
\hline N.A.S. & 3 & 2 & 2 & 2 & 3 & 2 & 2 & 2 & 2 & 2 & 2 & 2 & 3 & 2 & 2 & 1 \\
\hline N.A.M. & 3 & 2 & 2 & 1 & 2 & 2 & 1 & 2 & 1 & 2 & 2 & 2 & 2 & 2 & 1 & 2 \\
\hline N.I.A. & 5 & 5 & 5 & 4 & 5 & 5 & 4 & 3 & 6 & 5 & 4 & 3 & 6 & 6 & 4 & 4 \\
\hline N.I.A.H. & 7 & 7 & 6 & 7 & 7 & 7 & 6 & 6 & 7 & 7 & 7 & 8 & 7 & 8 & 7 & 7 \\
\hline TOTAUX & 18 & 16 & 15 & 14 & 17 & 16 & 13 & 13 & 16 & 16 & 15 & 15 & 18 & 18 & 14 & 14 \\
\hline
\end{tabular}

A :' plateau; B: haut de pente; C: pente; $D$ : bas-fond.

N.A.S: niveou arborescent supérieur:

N.A.M.: niveau arborescent moyen:

N.I.A.: niveau inférieur arbustif;

N.I.A.H.: niveau inférieur arbustif et herbacé. 
La position de plateau au Km. 22 se distingue par 2 hoplexols au lieu de 3 ce qui rend compte de l'absence d'un ensemble arborescent. Au Km. 38, la variation la plus importante apparait dans le relevé de bas-fond ou on ne note que 2 hoplexols, au dessus de $12 \mathrm{~m}$, mas caractérisés, en partie, par un monophyse traduisant l'importance des palmiers dans la voûte.

\section{CONCLUSIONS SUR LA STRUCTURE}

Sur 4 relevés de plateau, 3 présentent 3 ensembles arborescents de hauteur supérieure à $15 \mathrm{~m}, 1$ seul, au Km. 22, n'en posséde que 2 . Dans ce cas, l'analyse des hoplexols montre que le supérieur ( $\mathrm{n}^{\circ} 16$ entre 20 et $25 \mathrm{~m}$ ) réunit les feuillages d'un ensemble d'arbres du présent, paliphyse, et d'un ensemble d'arbres n'ayant pas atteint leur expansion maximale, propaliphyse, mais qui continueront de croltre jusqu' à dominer les précédents.

Les arbres et arbustes se trouvent à différents degrés de développement (prophyse. propaliphyse, paliphyse), ce que traduisent les variations dans les diagnoses.

Les feuiliages se distribuent de différentes manières; ainsi dans une grande clairière, les arbres disposent de l'énergie lumineuse maximale et se ramifient trés tôt. Leurs feuillages s'accroîtront en hauteur et participeront à plusieurs hoplexols, occupant alors tout l'espace vertical et ralentissant le développement des ensembles arborescents inférieurs.

C'est cette dynamique qui explique les variations entre relevés, principalement aux niveaux arborescents moyens.

\section{QUELQUES PARAMÈTRES QUANTITATIFS}

Aux mêmes endroits que furent faits les relevés morpho-structuraux, soit en 4 positions topographiques de 4 toposéquences, et sur des surfaces de $600 \mathrm{~m}^{2}(30 \times 20 \mathrm{~m})$, nous avons mesuré :

- la répartition par diamètres, mesurés à hauteur de poitrine, de 5 en $5 \mathrm{~cm}$ et à partir de $5 \mathrm{~cm}$, de tous les arbres, palmiers et lianes.

- le nombre total de palmiers d'une hauteur supérieure à $1 \mathrm{~m}$.
Les aires basales totales, la proportion représentée par les arbres d'un diamètre supérieur à $40 \mathrm{~cm}$ sont donnóes, ainsi que les mesures indiquée ci-dessus, dans les tableaux VI et VII.

II existe un rapport entre la faible importance des arbres de diamètre supérieur à $40 \mathrm{~cm}$ et le développement des ensembles arborescents au-dessus de $15 \mathrm{~m}$ :

-. plateau du Km. $22-2$ hoplexols au lieu de 3 et la plus faible valeur, $21 \%$, représentée par les diamètres supérieurs à $40 \mathrm{~cm}$ de tous les relevés de plateaux.

-- Haut de pente du Km. 22 - il y a bien 2 hoplexols comme dans les autres relevés, mais aucun arbre dont le diamètre soit égal ou supérieur à $40 \mathrm{~cm}$. Or l'hcplexol supérieur est uniquement constitué de feuillages d'arbres en cours de croissance (propaliphyse) comme, en partie, l'hoplexol immédiatement sousjacent.

- pente du Km. 22 - là aussi le faible pourcentage $(13 \%)$ représenté par les diamè tres à partir de $40 \mathrm{~cm}$ est lié à la présence d'arbres jeunes dont les feuillages encore incompiètement développés (propaliphyse) apparaissent dans l'hoplexol supérieur.

- - bas-fonds du Km. 38 - la plus faible valeur des 4 bas-fonds est iiée à l'absence d'un niveau arborescent au-dessus de $12 \mathrm{~m}$.

II esi évident qu'il y a des relations entre les données quantitatives et l'analyse morphostructurale. Notons cependant que la surface nécessaire à un relevé de ce iype est toujours supérieure à $600 \mathrm{~m}^{2}$, d'autre part que, avec une même aiagnose structurale, l'ımportance rela. tive des arbres de diamètre égal ou supérieur

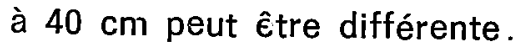

Les diamètres supérieurs à $40 \mathrm{~cm}$ ne sont pas très nombreux, ils sont même absents du haut de pente de la toposéquence du $\mathrm{Km} .22$, mais ils jouent un rô!e important dans la valeur totale de l'aire basale (plus de $50 \%$ sur la pente du $\mathrm{Km}$. 38). Les relevés n'étaient pas d'une taille suffisante pour donner une idée de la répartition des très gros diamètres; sur les 16 relevés seuls deux arbres dépassaient 70 $\mathrm{cm}$, l'un avec un diamètre entre 80 et $85 \mathrm{~cm}$ l'autre, tout à fait exceptionnel, de près de $2 \mathrm{~m}$. 
TABLEAU VI - Données quantitatives

\begin{tabular}{|c|c|c|c|c|c|c|c|c|c|c|c|c|c|c|c|c|}
\hline \multirow{2}{*}{$600 \mathrm{~m}^{2}$} & \multicolumn{4}{|c|}{ Km. 14} & \multicolumn{4}{|c|}{ Km. 20} & \multicolumn{4}{|c|}{ Km. 22} & \multicolumn{4}{|c|}{$\mathrm{Km} .38$} \\
\hline & A & B & c & D & A & B & C & D & A & B & C & D & A & B & C & D \\
\hline $\begin{array}{l}\text { Arbres, palmiers et lianes. } \\
\text { Diamètre } \geqslant 5 \mathrm{~cm} \text {. }\end{array}$ & 100 & 101 & 73 & 98 & 107 & 101 & 121 & 96 & 116 & 119 & 112 & 76 & 91 & 92 & 86 & 63 \\
\hline Aire basale en $\mathrm{m}^{2}$. & 2,9 & 2,1 & 2,7 & 2,2 & $2,6^{*}$ & 2,3 & 2,6 & 1,7 & 2,2 & 1,8 & 2,0 & 1,9 & 2,0 & 2,1 & $\Rightarrow, 0$ & 1,6 \\
\hline $\begin{array}{l}\text { Arbres. Diamètre } \geqslant 40 \mathrm{~cm} \text {. } \\
\% \text { aire basale totale } \\
\text { représentée par ces arbres }\end{array}$ & 6 & 2 & 3 & 3 & $5^{*}$ & 3 & 2 & 380 & 3 & - & 2 & 2 & 2 & 4 & 5 & 2 \\
\hline Palmiers. Alt. $>1 \mathrm{~m}$. & 76 & 74 & 56 & 68 & 57 & 78 & 58 & 119 & 71 & 74 & 55 & 114 & 40 & 51 & 36 & 92 \\
\hline $\begin{array}{l}\text { 1 ha } \\
\text { Nombre total } \mathrm{d} \text { ' individus. } \\
\text { diamètre } \geqslant 5 \mathrm{~cm} \text {. }\end{array}$ & 1666 & 1683 & 1216 & 1633 & 1783 & 1683 & 2016 & 1600 & 1933 & 1983 & 1867 & 1267 & 1501 & 1533 & $=33$ & 1050 \\
\hline Aire basale en $\mathrm{m}^{2}$. & 48 & 35 & 45 & 36 & 43 & 38 & 43 & 29 & 36 & 31 & 33 & 33 & 34 & 35 & 35 & 27 \\
\hline
\end{tabular}

- La présence d"un "angelim pedra", Dinizia excelso Ducke avec un diamètre de $2 \mathrm{~m}$ modifie profondément la valeur de l'aire basale qui atteint alors $5,6 \mathrm{~m} 2 / 600$ r 2 . II est évident qu'on ne peut tenir compte de la présence de cet arbre pour calculer la valeur de l'aire basale à l'hectare.

A: platau: $B$ : hou; de pente; $C:$ pentet $D:$ bosfond 
TABLEAU VII — Répartition en classes de diamètres des arbres, palmiers et lianes à partir de $5 \mathrm{~cm}$.

\begin{tabular}{|c|c|c|c|c|c|c|c|c|c|c|c|c|c|c|c|c|c|c|}
\hline \multirow{2}{*}{\multicolumn{3}{|c|}{$\theta \mathrm{cm}$}} & \multicolumn{4}{|c|}{$\mathrm{Km} 14$} & \multicolumn{4}{|c|}{$\mathrm{Km} .20$} & \multicolumn{4}{|c|}{$\mathrm{Km} .22$} & \multicolumn{4}{|c|}{ Km. 38} \\
\hline & & & A & B & C & D & A & B & C & D & A & B & C & D & A & B & $\mathrm{C}$ & D \\
\hline 5 & - & 9 & 54 & 48 & 38 & 62 & 57 & 42 & 73 & 64 & 65 & 63 & 66 & 27 & 54 & 54 & 53 & 28 \\
\hline 10 & - & 14 & 22 & 22 & 15 & 13 & 21 & 27 & 25 & 11 & 21 & 22 & 18 & 19 & 10 & 17 & 13 & 7 \\
\hline 15 & - & 19 & 4 & 12 & 7 & 9 & 9 & 15 & 7 & 10 & 11 & 14 & 8 & 17 & 12 & 7 & 5 & 12 \\
\hline 20 & - & 24 & 3 & 9 & 2 & 5 & 6 & 5 & 9 & 4 & 8 & 10 & 8 & 5 & 8 & 6 & 7 & 7 \\
\hline 25 & - & 29 & 6 & 2 & 2 & 2 & 5 & 4 & 3 & 3 & 5 & 5 & 6 & 2 & 1 & 2 & 2 & 2 \\
\hline 30 & - & 34 & 3 & 5 & 2 & 2 & 1 & 2 & 2 & & & 4 & 1 & 2 & 3 & 2 & & 3 \\
\hline 35 & - & 39 & 2 & 1 & 4 & 2 & 2 & 3 & & 1 & 3 & 1 & 3 & 2 & 1 & & 1 & 2 \\
\hline 40 & - & 44 & 2 & & & 1 & & 3 & 1 & & 2 & & 2 & & 1 & & 1 & 1 \\
\hline 45 & - & 49 & 1 & 1 & & 0 & 2 & & & 1 & 1 & & & & & 2 & 2 & 1 \\
\hline 50 & - & 54 & 1 & & 1 & 1 & 1 & & & 1 & & & & 1 & & & & \\
\hline 55 & - & 59 & & 1 & & & 1 & & 1 & 1 & & & & 1 & & 1 & 1 & \\
\hline 60 & - & 64 & 1 & & 1 & & 1 & & & & & & & & & 1 & 1 & \\
\hline 65 & - & 69 & 1 & & 1 & 1 & & & & & & & & & & & & \\
\hline 70 & - & 74 & & & & & & & & & & & & & & & & \\
\hline 80 & & 84 & & & & & & & & & & & & & & & & \\
\hline & & 199 & & & & & 1 & & & & & & & & 1 & & & \\
\hline & ТОT & $\mathrm{AL}$ & 100 & 101 & 73 & 98 & 107 & 101 & 121 & 96 & 116 & 119 & 122 & 76 & 91 & 92 & 86 & 63 \\
\hline
\end{tabular}

A: 'plateau; B: haut de pence; $C$ : pente; D: bas-fond. 


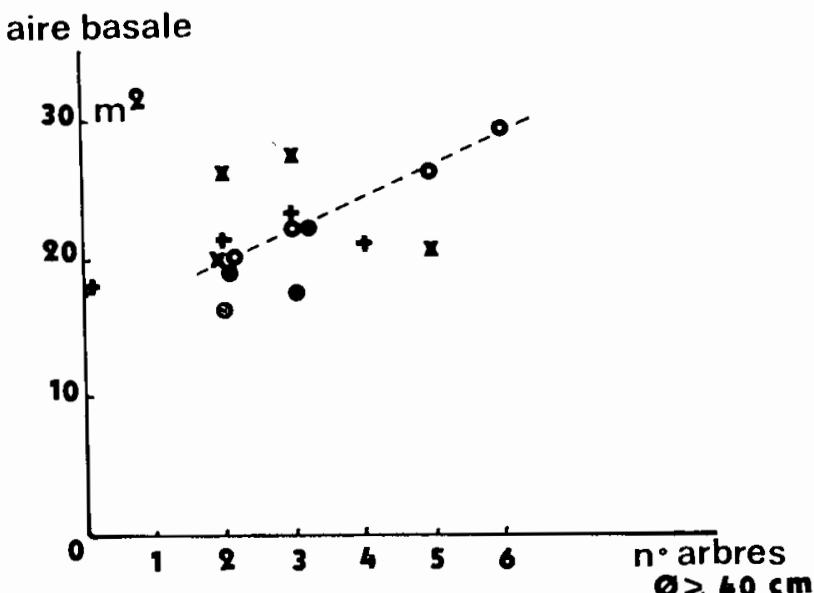

Fig. 3 - Relation entre la nombre d'arbres ayant un diamètre égal ou supérieur à $40 \mathrm{~cm}$ et l'aire basale sur $600 \mathrm{~m}^{2}$. o : plateaux; + : hauts de pente; $x$ : pentes; 0 : bas-fond. La droite en tiretés relie les 4 valeurs de plateau.

La variation de l'aire basale entre les 16 . relevés, si elle est considérable, n'atteint pas pour autant les valeurs constatées dans d'autres régions d'Amazonie ( $F$. Kahn, bassin du Tocantins) et se situe dans des limites raisonnables, de 1 à 1,6 , étant données les dimensions de l'échantillonago.

Les valeurs sur les hauts de pente et dans les bas-fonds osnt plutôt inférieures à celles sur plateaux et pentes (Tab. VIII); il y a une bonne relation avec le nombre de diamètres supéitieurs à $4 \mathrm{C} \mathrm{cm}$,et très bonne même poưr les 4 positions de plateaux (Fig. 3).

\section{CONCLUSION}

La comparaison de 4 toposéquences dans cette région comprise entre la route BR-174 et le Cuieiras témoigne d'une homogénéité structurale de végétation certaine.

La variabilite ne dépend pas de l'échantillonage relativement réduit mais essentiellement du relief, de la position topographique et du cycle silvigénétique. Cette variabilité est plutòt moins marquée que ce qui fut observé dans d'autres régions d'Amazonie.

Si la position topographique est importante, qu'elle conditionne des propriétés du sol ou que la déclivité joue le rôle majeur, c'est pourtant le processus d'auto-régénération de la forêt qui entraîne les variations structurales et provoque l'hetérogénéité.

La forêt de plateaux, ou mieux des sommets des "demi-oranges" qui caractérisent le paysage géomorphologique de la région étudiée, se révèle relativemerit homogène et marquée par la présence d'un ensemble supérieur d'arbres du présent, bien que peu développé, apparaissant comme un niveau d'émer. gents.

Les pentes dans leur ensemble sont plus hétérogènes. Les bas-fonds se séparent nette. ment du reste de la toposéquence, mais sont cependant très comparables entre eux.

TABLEAU VIII - Aire basale $\left(\mathrm{m}^{2} / \mathrm{ha}\right)$ en fonction de la position topographique et de la toposéquence.

\begin{tabular}{|c|c|c|c|c|c|}
\hline $\begin{array}{l}\text { Toposéquences } \\
\text { Position }\end{array}$ & $\mathrm{Km} .14$ & $\mathrm{Km} \cdot 20$ & Km. 22 & $\mathrm{Km} .38$ & Moyenne \\
\hline Plateau & 48 & 43 & 36 & 34 & 40,25 \\
\hline Haut de pente & 35 & 38 & 31 & 35 & 32,25 \\
\hline Pente & 45 & 43 & 33 & 35 & 39 \\
\hline Bas-fond & 36 & 29 & 33 & 27 & 31,25 \\
\hline Moyenne & 41,75 & 38,25 & 33,25 & 32,75 & $\begin{array}{c}\text { Moyenne genérale } \\
36,5\end{array}$ \\
\hline
\end{tabular}


On peut dire qu'il existe une réalité dans la structure de la végétation le long d'une même toposéquence, que cette réalité peut être décrite en termes qualitatifs et quantitatifs et qu'elle est représentative d'une région donnée (ici les bassins étudiés).

Le dynamisme interne de la forêt confère une héterogénéité locale qui est responsable de la structure en mosaïque souvent évoquée mais peu étudiée.

\section{Resumo}

Os autores fazem um estudo comparativo da estrutura da vegetação de duas toposseqüências nas bacias hidrográficas da ZF-02, segundo um método morfoestrutural do que recordam o essencial. Discutem as variações na toposseqüência e os fatores de variação. Comparam os resultados com duas outras toposseqüências. Alguns parâmetros quantitativos, diâmetro, área basal, são interpretados em função do ciclo silvigenético. Esta análise evidencia a homogeneidade da vegetaçăo florestal nesta área, e a existência de uma unidade estrutural da topossequêencia. $O$ principal fator de variação é o ciclo silvigenético, processo de auto-regeneração da floresta pela queda das árvores e a cica. trização das clareiras naturais.

\section{BIBLIOGRAPHIE}

COUSENS, I.L.

1965 - Some reflections on the nature of Malayan lowland rain forest. Malay. For., 28: 122128.

DENSLOW, J.S.

1980 - Gap partitioning among tropical rain forest trees. Biotropica, 12 (2): 47-55.
DESCOINGS, B.

1976 - Pour une conception structurale et ouverte des classifications phytogéographiques. Adansonia, 16: 93-105.

GUILLAUMET, J.L. \& KAHN, F.

1978 - Les diagnoses de la végétation. In: Recherche d'un langage transdiciplinaire pour l'étude du milieu naturel (Tropiques humides). Trav. et. doc. ORSTOM, 91: 43-53.

1979 - Description des végétations forestières tropicales, approche morphologique et structurale - Candollea, 34 (1): 109-131.

HALLE, F. \& OLDEMAN, R.A.A

1970 - Essai sur l'architecture et la dynamique de croissance des arbres tropicaux. Paris, Masson.

HALLE, F.; OLDEMAN, R.A.A. \& TOMLISON, P.B.

1978 - Tropical trees and forests. An architectural analysis. Springer Verlag, Berlin, Heidelberg, New York.

HARTSHORN, G.S.

1978 - Tree falls and tropical forest dynamic. In: Tropical trees as living systems. Tomlinson, P.B. e Zimmermann, M.H.

1980 - Neotropical forest dynamics. Biotropica, 12 (2): 23-30.

OLDEMAN, R.A.A.

1974 - L'architecture de la forêt guyanaise. Mém. ORSTOM, 73.

RICHARDS, P.W.

1 1952 - The tropical rain forest. Cambridge Univ. Press.

WHITMORE, T.C.

1978 - Gaps in the forest canopy. In: Tropical trees as living systems. Tomlinson, P.B. e Zimmermann, M.H. (ed). 639-655.

(Aceito para publicação em 24/08/82) 